\title{
Colposcopy services in the West Midlands region
}

\author{
C B J Woodman, J A Jordan
}

\begin{abstract}
A survey of all 72 consultant gynaecologists in the West Midlands region was carried out to determine their views on colposcopy services. All districts provided a colposcopy service and 47 consultants practised colposcopy. The consultants differed considerably in their views on criteria for referring women for investigation after smears. All but one thought that a positive smear result was an indication for immediate referral but whereas $\mathbf{5 5}$ thought that women with one or more inflammatory smear should be referred, 17 did not believe this to be necessary. Sixty seven consultants thought a preoperative colposcopic assessment desirable, but $10 \mathrm{had}$ to ration referrals because of limited resources. Four forms of treatment were used: 17 districts had a carbon dioxide laser, eight used low voltage diathermy loop excision, two had only a cold coagulator, and one used only cold cautery. There was widespread dissatisfaction with resources and about the increasing amount of time spent on the service at the expense of other work. Consultants were also concerned about the relevance of much of what was done to the aim of the screening programmereducing mortality from cervical cancer.

Although additional resources are needed in some districts, referral policies, indications for colposcopic assessment, training, choice of treatment, and the value of follow up need to be reviewed if the service is to be improved.
\end{abstract}

\section{Introduction}

Recently introduced call and recall services for cervical cytology have directed attention to the resources available for investigating and treating women who are found to have an abnormality. Gynaecologists and public health doctors in many districts are concerned that the existing services may not be able to cope with the increased numbers of women with suspected abnormalities who will be identified by the proposed screening programme. We carried out a survey of consultant gynaecologists to find out what resources were available to investigate and treat women with cytological abnormalities in each of the 22 districts in the West Midlands region.

Medicine, Medical School University of Birmingham, Edgbaston, Birmingham B15 2TJ

C B J Woodman, MFPHM, senior lecturer

\section{Birmingham Maternity} Hospital, Birmingham J A Jordan, FRCOG, consultant gynaecologist

Correspondence to:

Dr Woodman.

Br. Med. Y 1989;299:899-901

\section{Methods and results}

One consultant in each district known to have an interest in colposcopy and cervical pathology was asked for statistics relating to $(a)$ the number of new patients referred with abnormal cytology in the district in 1987; $(b)$ the workload of managing patients with cervical intraepithelial neoplasia; and $(c)$ the proportions of patients treated by different methods in 1987 .

All 72 consultant gynaecologists in the West Midlands Regional Health Authority were also asked to take part in a telephone survey about the colposcopy

\section{Questions that need to be answered about a colposcopy service}

- What are the optimum referral criteria for assessment by a specialist after the discovery of cytological abnormality?

- Should preoperative colposcopy be done always or only in specific age groups?

- What is the optimum number of trained colposcopists and what are the implications for training? Should clinical assistants be employed as colposcopists?

- Colposcopy should be done in a dedicated outpatient clinic: what are the implications for additional space, staff, equipment, etc?

- What is the most efficient method of local destructive treatment for cervical intraepithelial neoplasia for outpatients?

- Should patients treated for cervical intraepithelial neoplasia be followed up by regular colposcopic review or cytological examination alone? How long should follow up be hospital based?

service and were circulated with the interview schedule in advance. All took part in the survey. Two districts shared a common gynaecological service and therefore were considered as one unit when the results were aggregated by district.

\section{CRITERIA FOR REFERRAL}

The 72 consultants were asked what they considered to be acceptable criteria for referring patients to hospital. All but one thought that a woman whose smear gave a positive result should be referred promptly to hospital. Forty six thought that a woman with a suspicious result should be referred immediately; 25 thought referral could be postponed until the woman had had two suspicious results, and one suggested that referral should be delayed until there were three suspicious results.

Two consultants thought that when a report suggested inflammatory changes the patient should be referred immediately, 28 thought after two such results, and 25 after three, but 17 did not think that inflammatory changes ever indicated referral.

Sixty seven consultants thought that a preoperative colposcopic assessment was always desirable; five did not. Ten, however, had to ration referrals because resources were limited in their unit. Rationing, whether enforced or elective, was based on the age of the patient, the severity of the cytological abnormality, and the need to preserve fertility. Thus some consultants elected to carry out a cone biopsy in postmenopausal women whose smear gave a positive result, while others would carry out a cone biopsy in women aged 35 and over. 
TABLE I - Number of consultants and proportion of colposcopy workload undertaken

\begin{tabular}{cc}
\hline $\begin{array}{c}\text { Proportion of } \\
\text { workload }(\%) \\
\text { personally } \\
\text { undertaken }\end{array}$ & $\begin{array}{c}\text { No of } \\
\text { consultants } \\
(\mathrm{n}=47)\end{array}$ \\
\hline 100 & 25 \\
$75-90$ & 10 \\
$50-74$ & 7 \\
$<50$ & 5
\end{tabular}

TABLE II -Waiting times for 38 outpatient colposcopy clinics

\begin{tabular}{lc}
\hline No of weeks & $\begin{array}{c}\text { No of consultant } \\
\text { practices }\end{array}$ \\
\hline 02 & 6 \\
$3-5$ & 17 \\
$6-8$ & 5 \\
$9-11$ & 2 \\
$12-14$ & 6 \\
$15-17$ & 1 \\
48 & 1 \\
\hline
\end{tabular}

COLPOSCOPY SERVICE

All 22 districts provided a colposcopy service. Twenty offered treatment to outpatients. Forty seven of the 72 consultant gynaecologists practised colposcopy. They were helped by one associate specialist, seven clinical assistants, three research fellows, and the junior medical staff. In two districts a clinical assistant provided the colposcopy service and in one a registrar in obstetrics and gynaecology.

Each of the 47 consultants who practised colposcopy was asked to estimate the proportion of the colposcopy workload that he or she personally undertook. Table I shows that in most cases consultants provided the service, with junior medical staff only occasionally contributing.

Thirty eight of the 47 consultant colposcopists practised in a dedicated outpatient clinic and four in their general gynaecology clinics. Five had to use operating theatre sessions. Table II shows the waiting time for outpatient appointments in the colposcopy clinic according to consultant practice.

Five districts reported only on the number of new patients referred for colposcopy, four on the relative frequency with which cervical intraepithelial neoplasia of differing severity was found on biopsy material, and three on how often different methods of treatment were used. Two consultants working in two other districts provided this information for their patients. Of the 1505 patients for whom information was available, $1255(83 \%)$ were treated by local destructive methods, $202(13 \%)$ by cone biopsy, and $48(3 \%)$ by hysterectomy.

Treatment-Four different treatments were offered to outpatients in the region. Seventeen districts had a dedicated carbon dioxide laser. In 10 districts the laser had been bought from funds generated from public appeals, in five it was bought by the district health authority, and in two funding was obtained from both sources. The cold coagulator was the only method of treatment used in two districts and cryocautery the only method used in one other district. Twelve consultants used both the carbon dioxide laser and cryocautery or cold coagulation to treat cervical intraepithelial neoplasia, using cold coagulation to remove minor dysplastic lesions. Low voltage diathermy loop excision' is a new treatment that was available in eight districts and used by 13 consultants, for six of whom it had become the preferred treatment. A further two districts had placed orders for the loop diathermy machine, and others were considering using this treatment.

Follow up-Twenty one of the 47 consultant colposcopists reviewed the cases of all the women treated in the colposcopy clinic, while two reviewed only those treated for cervical intraepithelial neoplasia grade III. The median number of follow up visits prescribed under the different regimens was one (range one to four). Some consultants arranged additional follow up visits for patients with cervical intraepithelial neoplasia grade III lesions. Twenty four used cytological examination alone to assess outcome; the smears were usually taken in the general gynaecology clinics. The duration of follow up in hospital outpatient clinics prescribed by different consultants, including both colposcopic and cytological assessments, ranged from three months to 10 years. Ten consultants considered one follow up assessment to be sufficient, 28 consultants two, and nine consultants three or more. Five consultants thought that women treated for cervical intraepithelial neoplasia grade III should have a longer hospital follow up.

Adequacy of resources - The consensus in eight districts was that their colposcopy service was unsatisfactory, and consultants in all districts were dissatisfied with resources-particularly time, staff, and equip- ment. Eighteen districts wanted more colposcopy sessions. The need for additional nursing and clinical support and also the effect that an increase in the colposcopy service would have on theatre use were recognised. In four districts consultants thought that they already had sufficient medical staff if outpatient time and nursing and clerical support were available. The general opinion in the remaining districts was that the increase in workload would require new staff. A clinical assistant was preferred in most districts, although in two districts a consultant gynaecologist with an interest in colposcopy was thought to be essential; one consultant suggested using nurse practitioners. Two districts would welcome another colposcope, two that did not have a laser wanted one, in two the laser needed replacing, and one was attempting to acquire a loop diathermy machine.

\section{Discussion}

The secondary prevention of cervical cancer requires an effective cytology screening programme and an efficient method of treating women who are found to have abnormalities. The objective of screening is to reduce the number of deaths from cervical cancer, but the immediate requirement as recognised by clinicians is to restore cytological normality in women who are referred with abnormal smear results. The development of colposcopy has made this easier. The results of our survey show that colposcopy services in the West Midlands face many problems and inconsistencies. There is no reason to believe that these findings are not typical of services in other regions.

The workload for colposcopy is determined by the number of women with abnormal smear results who are referred for investigation in hospital, which in turn is a function not only of the prevalence of the disease but also of the opinions of those who carry out cytology screening and the referral policies of those who take smears. Some doctors think that those who carry out screening often err on the side of calling women back who have doubtful abnormalities through fearing to make mistakes owing to inadequate experience or supervision. Many screeners have not had formal training, and continuing education programmes are the exception rather than the rule, though steps are being taken to rectify such omissions.

Referral policies influence colposcopy workload directly. Laboratories usually make firm recommendations to general practitioners about the need for hospital referral, and it is not surprising if general practitioners, who may not understand completely the clinical course of the disease, choose to accept the advice. Some consultants are resentful of the firm recommendations for referral made on smear reports, but it is easy to sympathise with laboratory staff, who are anxious to ensure that appropriate action is taken, knowing that the knowledge among smear takers varies.

These difficulties could be resolved if responsibilities were clearly defined and there were explicit guidelines for referral. This requires discussion among colposcopists, general practitioners, and laboratory staff and agreement on the criteria for referral. The divergence of opinion in our survey on the need to refer women with inflammatory changes suggests that this will not be easily achieved.

Most consultants in our survey thought that preoperative assessment by colposcopy was always desirable, but some had to ration referrals because of limited resources. The costs and benefits of a policy where only selected patients are referred for colposcopy need to be evaluated. The costs are those incurred when a woman who can be treated as an outpatient has an unnecessarily radical treatment: the benefits arise from 
reducing the number of assessments that do not influence patient management. The results of a recent inquiry showed that colposcopic assessment of postmenopausal women has limited value. ${ }^{3}$ Thus there is reason to suspect that this cost-benefit equation will vary across different age groups, and this merits study.

Although two thirds of the 72 consultant gynaecologists in the region practised colposcopy, the steady increase in workload placed a strain on the colposcopy service and affected other aspects of gynaecological practice. In most cases the colposcopy service was provided entirely by consultants. Some districts appointed clinical assistants to relieve this strain and others seek to do so. Although it may not be desirable for every consultant gynaecologist to practise colposcopy, it seems reasonable that sufficient numbers should be trained to provide a minimum of one consultant colposcopist for each district. The increasing use of clinical assistants may be necessary and inevitable, but we think that the valuable contribution made by these practitioners is optimal when they are located in a unit that includes a consultant colposcopist.

The principal advantage of a colposcopy service is that it enables patients to be selected for more conservative treatment. There is a further benefit if this can be offered in the outpatient department. When there is no facility for outpatient treatment the full potential of the service is not realised. One district in our survey did not yet offer outpatient treatment, and some consultants in other districts were obliged to use theatre sessions for assessment and treatment. This is not only suboptimal practice but an irrational use of resources.

When most units are looking for additional resources because of increasing workloads it is disappointing that so few could describe their throughput by the number of new referrals, number of patients treated, and type of treatments given. Although information about treatment was available for about 1500 patients, there are problems in interpreting these data because of the difficulty in linking biopsy specimens and treatments to individual patients, and because of the certainty that some patients had more than one biopsy and more than one treatment episode. There is an urgent need for reliable activity analysis.

While some districts were taking possession of their first carbon dioxide laser or were considering a replacement consultants in other districts were concluding that loop excision, which is cheaper and provides a useful increase in the size of the biopsy specimen, was the preferred treatment. There seems little justification in spending money on dedicated laser equipment to treat outpatients until loop diathermy has been fully evaluated.

There was no general agreement about length, type, and frequency of follow up after treatment for cervical intraepithelial neoplasia. Colposcopic follow up is generally advised, but pressure on the service usually precludes regular long term review, even if this were shown to be valuable. Colposcopic follow up probably brings forward the time at which residual disease can be detected, but it is less certain whether it reduces the number of cancers that eventually occur. The incidence of invasive cancer after laser treatment is so low that a large randomised trial of different follow up regimens would be needed to show a substantial difference. The development of the loop excision method, with its greater certainty of finding an abnormality, may anyway make these calculations irrelevant. It is irrational to insist on a follow up regimen for which there is little supporting evidence when most practitioners find it impracticable.

A service that efficiently treats women with abnormal smear results may still fail to reduce mortality from cervical cancer. There is already evidence that a substantial proportion of women fail to attend for a smear when invited to do so and that only a large increase in resources will ensure a more acceptable coverage. Thus it may be difficult to sustain a hospital service that offers immediate assessment for minor cytological abnormalities.

The benefits arising from the investigation and treatment of women with abnormal cytology are related to the number of deaths avoided and cancers prevented, and the costs of this activity are related to the number of patients in which treatment is unnecessary. The balance between costs and benefits is determined in part by the referral threshold. The more women with minor abnormalities who are referred the more unnecessary investigation and treatment is undertaken. It is often argued that no valid threshold exists as a small proportion of women with minor abnormalities have cervical intraepithelial neoplasia grade III and therefore all should be referred. ${ }^{45}$ What should be considered, but rarely is, is the likelihood of these women proceeding to invasive carcinoma without progressing through the more severe degrees of cytological abnormality. It is the responsibility of those with the greatest understanding of the clinical course of the disease to set the criteria for referral, which must take account of the constraints imposed by limited resources as well as reflect the primary objective of reducing mortality from cervical carcinoma.

We thank the consultant gynaecologists in the West Midlands region for their cooperation, many constructive comments, and valuable insights. We thank Mrs $M$ Whitlock for help in preparing the paper.

1 Prenderville W, Cullimore JE. Low voltage diathermy loop excision of the transformation zone-a new treatment for CIN. Colposcopy and Gynaecological Laser Surgery 1988;3:232.

2 Department of Health and Social Security. Advance letter (PTB) July 1988 London: DHSS.

3 Constantine G, Williams DR, Leusley DM. The management of postmenopausal women with abnormal cytology. Colposcopy and Gynaecological Laser Surgery 1987;3:93-7.

4 Soutter WP, Wisdom S, Broughs AK, Monaghan JM. Should patients with mild atypia in a cervical smear be referred for colposcopy? $\mathrm{Br} \mathcal{F}$ Obstet mild atypia in a cervical
Gynaecol 1986;93:70-4.

5 Campion MJ, Singer A, Mitchell HS. Complacency in diagnosis of cervical cancer. Br Med F 1987;294:1337-40.

(Accepted 23 fune 1989)

\section{ANY QUESTIONS}

Is gynaecomastia ever associated with adenocarcinoma of the prostate?

Gynaecomastia is not caused by carcinoma of the prostate but may occur as a side effect of its treatment. Stilboestrol and other oestrogenic compounds used in managing prostatic cancer predictably cause gynaecomastia. Cyproterone acetate, a progestogen with antiandrogenic activity also commonly used in the treatment of prostatic cancer, may also cause gynaecomastia but only in about $15 \%$ of patients. Generally the gynaecomastia is caused by the drugs producing hyperprolactinaemia. Cimetidine, digoxin, ethionamide, griseofulvin, isoniazid, and spironolactone are the more common drugs implicated.

Gynaecomastia in the absence of treatment requires a search for hormone secreting tumours of the pituitary or testes. Magnetic resonance imaging or computed tomography of the pituitary fossa may be required in the first case and careful clinical examination and ultrasound scanning of the testes in the second. Gynaecomastia may occur in liver failure. In unilateral breast enlargement the possibility of a primary tumour of the breast must be considered and excluded by careful palpation and biopsy if necessary.-J G GINGELL, consultant urologist, Bristol 